\title{
Pigs vs people: the use of pigs as analogues for humans in forensic entomology and taphonomy research
}

\author{
Szymon Matuszewski ${ }^{1}$ (D) Martin J. R. Hall ${ }^{2} \cdot{\text { Gaétan } \text { Moreau }^{3} \cdot \text { Kenneth G. Schoenly }}^{4} \cdot$ Aaron M. Tarone $^{5}$. \\ Martin H. Villet ${ }^{6}$
}

Received: 31 October 2018 / Revised: 2 May 2019 / Accepted: 17 May 2019/Published online: 17 June 2019

(C) The Author(s) 2019

\begin{abstract}
Most studies of decomposition in forensic entomology and taphonomy have used non-human cadavers. Following the recommendation of using domestic pig cadavers as analogues for humans in forensic entomology in the 1980s, pigs became the most frequently used model cadavers in forensic sciences. They have shaped our understanding of how large vertebrate cadavers decompose in, for example, various environments, seasons and after various ante- or postmortem cadaver modifications. They have also been used to demonstrate the feasibility of several new or well-established forensic techniques. The advent of outdoor human taphonomy facilities enabled experimental comparisons of decomposition between pig and human cadavers. Recent comparisons challenged the pig-as-analogue claim in entomology and taphonomy research. In this review, we discuss in a broad methodological context the advantages and disadvantages of pig and human cadavers for forensic research and rebut the critique of pigs as analogues for humans. We conclude that experiments using human cadaver analogues (i.e. pig carcasses) are easier to replicate and more practical for controlling confounding factors than studies based solely on humans and, therefore, are likely to remain our primary epistemic source of forensic knowledge for the immediate future. We supplement these considerations with new guidelines for model cadaver choice in forensic science research.
\end{abstract}

Keywords Forensic entomology · Forensic taphonomy · Pig carcasses · Human corpses · Animalmodels · Decomposition ecology

Electronic supplementary material The online version of this article (https://doi.org/10.1007/s00414-019-02074-5) contains supplementary material, which is available to authorized users.

Szymon Matuszewski

szymmat@amu.edu.pl

1 Laboratory of Criminalistics, Adam Mickiewicz University, Św. Marcin 90, 61-809 Poznań, Poland

2 Department of Life Sciences, Natural History Museum, Cromwell Road, London SW7 5BD, UK

3 Département de biologie, Pavillon Rémi-Rossignol, Université de Moncton, Moncton, New Brunswick E1A 3E9, Canada

4 Department of Biological Sciences, California State University, Stanislaus, 1 University Circle, Turlock, CA 95382, USA

5 Department of Entomology, Texas A\&M University, 2475 TAMU, College Station, TX 77843, USA

6 Southern African Forensic Entomology Research Laboratory, Rhodes University, Makandha 6140, South Africa
"We are unlikely to ever know everything about every organism. Therefore, we should agree on some convenient organism(s) to study in great depth, so that we can use the experience of the past (in that organism) to build on in the future. This will lead to a body of knowledge in that 'model system' that allows us to design appropriate studies of nonmodel systems to answer important questions about their biology" [1].

"Model species are usually easy to rear, observe, or otherwise experimentally manipulate. They therefore allow knowledge to be built up rapidly and efficiently, because confounding factors are known and thus can be controlled in subsequent experiments" [2].

\section{Introduction}

While collaborating with medical examiners in the late 1800s, French entomologist Pierre Mégnin [3] advanced the first 
formal definition and testable mechanism of ecological succession and recognized the predictability of carrion-arthropod succession and resource partitioning in human corpses and their application in forensic analysis $[4,5]$. These investigations gave birth to the twin disciplines of carrion ecology and forensic entomology. Subsequently, most studies of vertebrate decomposition used non-human carcasses ranging in size from amphibians to elephants (Table 1). Payne innovatively used pig cadavers in his ground-breaking ecological experiments on decomposition [6-8]. Wider interest in forensic entomology and taphonomy arose in the mid-1980s, and such studies initially focussed on pigs or rabbits (Table 1). By the late 1980 s, the domestic pig was being recommended as an analogue for humans in forensic entomology research and training workshops [9-11]. Starting in the early 1990s, field studies and statistical models were proposed to test different aspects of the pig-as-analogue claim in forensic entomology [12-14].

Examples of taphonomic studies have been cited from as far back as Leonardo da Vinci in the fifteenth century, but the field began to achieve formality in the 1940s [15]. In the 1970s, palaeoanthropology used taphonomy to interpret the deposition of hominid remains in fossil-rich sites, particularly to provide information about how the hominids lived and died $[16,17]$. Integration of fossil-focused taphonomy with physical anthropology led to the differentiation of forensic taphonomy, which relied on extensive comparisons of palaeontological, archaeological and modern case studies [18]. The development of pigs as model organisms in forensic entomology provided a more experimental approach for forensic taphonomy and established some major patterns regarding vertebrate decomposition (Table 1, Fig. 1).

The advent of outdoor human taphonomy facilities (often mistermed "body farms" [19]) facilitated experimental studies using human cadavers. First amongst these was the University of Tennessee Anthropological Research Facility, while the first outside the USA was the Australian Facility for Taphonomic Experimental Research (AFTER) [19, 20]. At least eight facilities now exist, six in the USA, one in Australia and one in the Netherlands [20-22]. The facilities have allowed experimental comparison of decomposition in human and non-human models under a variety of conditions $[14,23,24]$. Since then, debate has arisen over the relevance of taphonomic studies for forensics (e.g. [19, 25, $26]$ ), and the proper associated experimental (and ethical) protocols $[27,28]$. There is variation in the source populations contributing to taphonomy facilities; moreover, their source cadavers (usually elders dying of natural causes) systematically differ from cadavers involved in forensic scenarios (usually adults dying of unnatural causes). Therefore, for a variety of reasons, the findings from these facilities may be difficult to extrapolate to other human populations and to typical forensic cases.
Recent publications have raised the opportunity to consolidate what has been learned from animal models in decomposition studies, and to examine the implications of this knowledge for the design of field experiments in forensic entomology and taphonomy, specifically, whether animal carcasses can effectively substitute for human cadavers, which is the major aim of this review. Our major focus is research on principles concerning cadaver decomposition, including the associated arthropods and their succession. Therefore this paper does not extensively address topics related to the accuracy and precision of PMI estimation techniques developed in forensic entomology or taphonomy.

\section{Lessons from pig cadavers}

The use of animal models to advance knowledge dates back to the ancient Greek times with dogs and chicks used to study human anatomy, physiology and ontogeny [29]. Nowadays, animal models are used to study a large array of human related-issues, e.g. diseases [30], mental and neuropsychiatric disorders [31] or orthopaedic and dental implants [32]. In a similar way, our current understanding of animal decomposition is largely derived from experiments with non-human cadavers, with pig carcasses contributing overwhelmingly to this knowledge (Table 1). Payne's [6] experimental work using piglets was a watershed event in carrion ecology for its impact and originality. After trying carrion from different vertebrate animals (amphibians, mammals, birds), Payne settled on domesticated pigs because he knew the time of their death, he could acquire them in large numbers of uniform age and mass, and their relatively hairless skin and lack of feathers made insect sampling easier than from alternative carcasses. In his experiments, Payne used cages with different mesh sizes to provide open and limited access to insects to document daily changes in carcass decay and dismemberment. He found that carcasses protected from insects mummified, remaining intact for months; whereas, carcasses exposed to insects lost $90 \%$ of their starting mass in just 6 days. This result showed that insect access is a key determinant of cadaver decay.

Inspired by Payne's experimental protocol, forensic entomologists started using pig cadavers in studies focused on inventorying carrion-arthropod faunas and successional patterns, which have been described for a long list of countries and habitats (Table 1). Although the species involved varied between biogeographical regions, ecological guilds were consistent and functioned in a very consistent way (Table 1). Pigs have illustrated patterns of decomposition over timescales of days, seasons and years (Table 1). Seasonal components of variation in the insect community are relatively well understood and several quantitative models have been proposed to describe the ecological succession that occurs in the arthropod community on a cadaver (Table 1). Much of the early work 
Table 1 Selected cadaver studies in carrion ecology, forensic entomology and taphonomy. References to this table are listed in Electronic Supplementary Material

\begin{tabular}{|c|c|c|c|c|}
\hline Author(s) & $\begin{array}{l}\text { Date of } \\
\text { publication }\end{array}$ & Locality & Animal model & Major research focus \\
\hline Chapman and Sankey [1] & 1955 & England & Rabbits & Arthropod inventory; habitats \\
\hline Bornemissza [2] & 1957 & Australia & Guinea pig & Arthropod inventory; succession \\
\hline Reed [3] & 1958 & USA & Dogs & Arthropod inventory; succession \\
\hline Payne [4] & 1965 & USA & Pigs & Surface decomposition; insect access \\
\hline Payne et al. [5] & 1968 & USA & Pigs & Underground decomposition \\
\hline Payne and King [6] & 1972 & USA & Pigs & Water decomposition \\
\hline Nabagło [7] & 1973 & Poland & Bank voles & $\begin{array}{l}\text { Surface/underground decomposition; insect } \\
\text { inventory; succession; seasons }\end{array}$ \\
\hline Cornaby $[8]$ & 1974 & Costa Rica & Lizards, toads & Arthropod inventory; succession; habitats \\
\hline Johnson [9] & 1975 & USA & Small mammals & Arthropod inventory; succession; seasons \\
\hline Smith [10] & 1975 & England & Fox & Arthropod inventory; succession \\
\hline Coe [11] & 1978 & Kenya & Elephants & Surface decomposition; insect inventory \\
\hline McKinnerney [12] & 1978 & USA & Rabbits & Arthropod inventory; succession; scavenging \\
\hline Jiron and Cartin [13] & 1981 & Costa Rica & Dogs & Arthropod inventory; succession \\
\hline Abell et al. [14] & 1982 & USA & Turtles & Arthropod inventory; succession \\
\hline Rodriguez and Bass [15] & 1983 & USA & Humans & Insect inventory; succession \\
\hline Schoenly and Reid [16] & 1983 & USA & $\begin{array}{l}\text { Various } \\
\text { mammals }\end{array}$ & Cadaver mass; insect inventory \\
\hline Lord and Burger [17] & 1984 & USA & Gulls & $\begin{array}{l}\text { Arthropod inventory; succession; seasons; } \\
\text { habitats; scavenging }\end{array}$ \\
\hline Rodriguez and Bass [18] & 1985 & USA & Humans & Underground decomposition \\
\hline Early and Goff [19] & 1986 & Hawaii & Cats & Surface decomposition; arthropod inventory; succession \\
\hline Micozzi [20] & 1986 & USA & Rats & Freezing; wounds \\
\hline Braack [21] & 1986 & $\begin{array}{l}\text { South } \\
\text { Africa }\end{array}$ & Impala & Insect inventory \\
\hline Peschke et al. [22] & 1987 & Germany & Rabbits & Insect inventory; succession; habitats; seasons \\
\hline Tullis and Goff [23] & 1987 & Hawaii & Pig & Surface decomposition; arthropod inventory; succession \\
\hline Blacklith and Blacklith [24] & 1990 & Ireland & Birds, mice & Insect inventory; habitats \\
\hline Kentner and Streit [25] & 1990 & Germany & Rats & Insect inventory; succession; habitats \\
\hline Hewadikaram and Goff [26] & 1991 & Hawaii & Pigs & Cadaver mass \\
\hline Vass et al. [27] & 1992 & USA & Humans & Compounds released into soil during decomposition \\
\hline Shean et al. [28] & 1993 & USA & Pigs & Sun exposure \\
\hline Anderson and VanLaerhoven [29] & 1996 & Canada & Pigs & Insect inventory; succession \\
\hline Tantawi et al. [30] & 1996 & Egypt & Rabbits & Insect inventory; succession; seasons \\
\hline Keiper et al. [31] & 1997 & USA & Rats & Water decomposition; habitats; arthropod inventory \\
\hline Richards and Goff [32] & 1997 & Hawaii & Pigs & Arthropod inventory; succession; habitats \\
\hline Avila and Goff [33] & 1998 & Hawaii & Pigs & Burnt cadaver decomposition; habitats; succession \\
\hline Komar and Beattie $[34,35]$ & 1998 & Canada & Pigs & Cadaver mass; habitats; clothing; post-mortem artefacts \\
\hline Tomberlin and Adler [36] & 1998 & USA & Rats & Water decomposition; insect inventory; seasons; habitats \\
\hline Bourel et al. [37] & 1999 & France & Rabbits & Insect inventory; succession; habitats \\
\hline DeJong and Chadwick [38] & 1999 & USA & Rabbits & Insect inventory; succession; habitats \\
\hline Turner and Wiltshire [39] & 1999 & England & Pigs & Underground decomposition \\
\hline VanLaerhoven and Anderson [40] & 1999 & Canada & Pigs & $\begin{array}{l}\text { Underground decomposition; insect inventory; } \\
\text { succession; habitats }\end{array}$ \\
\hline Carvalho et al. [41] & 2000 & Brazil & Pigs, humans & Insect inventory \\
\hline Davis and Goff [42] & 2000 & Hawaii & Pigs & Intertidal habitats; succession \\
\hline Shalaby et al. [43] & 2000 & Hawaii & Pigs & Hanging cadaver decomposition; succession \\
\hline Arnaldos et al. [44] & 2001 & Spain & Chickens & Insect inventory; succession \\
\hline Carvalho and Linhares [45] & 2001 & Brazil & Pigs & Insect inventory; succession \\
\hline Marchenko [46] & 2001 & Russia & $\begin{array}{l}\text { Dogs, cats, } \\
\text { rabbits, pigs }\end{array}$ & $\begin{array}{l}\text { Decomposition in various scenarios; seasons; habitats; } \\
\text { insect repellents; clothing, plant response to cadavers }\end{array}$ \\
\hline Wolff et al. [47] & 2001 & Colombia & Pigs & Insect inventory; succession \\
\hline Yan et al. [48] & 2001 & USA & Pigs & Adipocere formation \\
\hline Centeno et al. [49] & 2002 & Argentina & Pigs & Insect inventory; seasons; habitats; succession \\
\hline Hobischak and Anderson [50] & 2002 & Canada & Pigs & Water decomposition; habitat; arthropod inventory; succession \\
\hline LeBlanc and Strongman [51] & 2002 & Canada & Pigs & Insect inventory; habitats \\
\hline Archer and Elgar $[52,53]$ & 2003 & Australia & Pigs & Insect inventory; seasons; colonisation patterns \\
\hline Bharti and Singh [54] & 2003 & India & Rabbits & Insect inventory; seasons; succession \\
\hline Kočárek [55] & 2003 & $\begin{array}{l}\text { Czech } \\
\text { Republic }\end{array}$ & Rats & Insect inventory; seasons; habitats; succession \\
\hline Shahid et al. [56] & 2003 & USA & Pigs & Arthropod saturation in human taphonomy facilities \\
\hline
\end{tabular}


Table 1 (continued)

\begin{tabular}{|c|c|c|c|c|}
\hline Author(s) & $\begin{array}{l}\text { Date of } \\
\text { publication }\end{array}$ & Locality & Animal model & Major research focus \\
\hline Watson and Carlton [57-59] & 2003,2005 & USA & $\begin{array}{l}\text { Bear, deer, } \\
\text { alligators, } \\
\text { pigs }\end{array}$ & $\begin{array}{l}\text { Insect inventory; seasons; succession; } \\
\text { animal models comparison }\end{array}$ \\
\hline Anderson and Hobischak [60] & 2004 & Canada & Pigs & Marine decomposition \\
\hline Archer $[61,62]$ & 2004 & Australia & Pigs & $\begin{array}{l}\text { Succession; seasons; annual variation; abiotic } \\
\text { determinants of decomposition rate }\end{array}$ \\
\hline Arnaldos et al. [63] & 2004 & Spain & Chickens & Insect inventory; seasons; succession \\
\hline Grassberger and Frank [64] & 2004 & Austria & Pigs & Urban decomposition; insect inventory; succession \\
\hline Tabor et al. $[65,66]$ & 2004,2005 & USA & Pigs & Insect inventory; succession; seasons \\
\hline Vass et al. [67] & 2004 & USA & Humans & Volatiles of decomposition \\
\hline Anderson [68] & 2005 & Canada & Pigs & Arson and insect evidence \\
\hline Moura et al. [69] & 2005 & Brazil & Rats & Succession mechanisms; seasons; habitats \\
\hline Perez et al. [70] & 2005 & Colombia & Pigs & Urban decomposition; insect inventory; succession \\
\hline Schoenly et al. [71] & 2005 & USA & Pigs & Arthropod saturation in human taphonomy facilities \\
\hline Weitzel [72] & 2005 & Canada & Pigs & Underground decomposition; seasons \\
\hline $\begin{array}{l}\text { DeJong and Hoback [73]; } \\
\text { DeJong et al. [74] }\end{array}$ & $2006 ; 2011$ & USA & Rats & Investigator disturbance; insect inventory; succession \\
\hline Hobischak et al. [75] & 2006 & Canada & Pigs & Sun exposure; insect inventory; succession \\
\hline Joy et al. [76] & 2006 & USA & Pigs & $\begin{array}{l}\text { Blow fly inventory; habitats; annual variation; } \\
\text { maggot mass }\end{array}$ \\
\hline Lang et al. [77] & 2006 & Australia & Possums & Insect inventory; colonisation patterns \\
\hline Adlam and Simmons [78] & 2007 & UK & Rabbits & Repeated cadaver disturbance \\
\hline Gruner et al. [79] & 2007 & USA & Pigs & Blow fly inventory; seasons; annual variation \\
\hline Martinez et al. [80] & 2007 & Colombia & Pigs & Insect inventory; succession \\
\hline O'Brien et al. [81] & 2007 & Australia & Pigs & Scavenging \\
\hline Schoenly et al. [82] & 2007 & USA & Pigs, humans & Sampling techniques; human/pig comparison \\
\hline Benninger et al. [83] & 2008 & Canada & Pigs & Compounds released into soil during decomposition \\
\hline Eberhardt and Elliot [84] & 2008 & $\begin{array}{l}\text { New } \\
\quad \text { Zealand }\end{array}$ & Pigs & Insect inventory; succession; habitats \\
\hline Fiedler et al. [85] & 2008 & Germany & Pigs & Adult fly inventory; succession; habitats \\
\hline Huntington et al. [86] & 2008 & USA & Pigs & Blow fly multigenerational colonisation \\
\hline Matuszewski et al. [87] & 2008 & Poland & Pigs & Insect inventory; succession; habitats \\
\hline Moretti et al. [88] & 2008 & Brazil & Mice, rats & Insect inventory; succession; seasons \\
\hline Sharanowski et al. [89] & 2008 & Canada & Pigs & Insect inventory; succession; seasons; sun exposure \\
\hline Ururahy-Rodrigues et al. [90] & 2008 & Brazil & Pigs & Post-mortem artefacts \\
\hline Voss et al. [91] & 2008 & Australia & Pigs & Inside-car decomposition; colonisation patterns \\
\hline Wang et al. [92] & 2008 & China & Pigs & Insect inventory; succession; seasons \\
\hline Charabidze et al. [93] & 2009 & France & Rats, Mice & Insect repellents; colonisation patterns \\
\hline Dekeirsschieter et al. [94] & 2009 & Belgium & Pigs & Volatiles of decomposition \\
\hline Kalinová et al. [95] & 2009 & $\begin{array}{l}\text { Czech } \\
\text { Republic }\end{array}$ & Mice & Carrion beetle attractants \\
\hline Kelly et al. $[96,97]$ & 2009,2011 & $\begin{array}{l}\text { South } \\
\text { Africa }\end{array}$ & Pigs & Wounds; wrapping; clothing \\
\hline Kjorlien et al. [98] & 2009 & Canada & Pigs & Scavenging; habitats; clothing \\
\hline Nelder et al. [99] & 2009 & USA & Alligators & Succession \\
\hline Özdemir and Sert [100] & 2009 & Turkey & Pigs & Insect inventory; succession; seasons \\
\hline Pakosh and Rogers [101] & 2009 & Canada & Pigs (limbs) & Water decomposition; \\
\hline Parmenter and MacMahon [102] & 2009 & USA & $\begin{array}{l}\text { Various } \\
\text { mammals } \\
\text { and birds }\end{array}$ & $\begin{array}{l}\text { Seasons; surface/underground decomposition; } \\
\text { scavenging; nutrient cycling }\end{array}$ \\
\hline Segura et al. [103] & 2009 & Colombia & Pigs & Insect inventory; succession \\
\hline Van Belle et al. [104] & 2009 & Canada & Pigs & $\begin{array}{l}\text { Compounds released into soil during decomposition; } \\
\text { surface/underground decomposition }\end{array}$ \\
\hline Voss et al. [105] & 2009 & Australia & Pigs & Insect inventory; succession; seasons; habitats \\
\hline Bachmann and Simmons [106] & 2010 & UK & Rabbits & Underground decomposition; colonisation patterns \\
\hline $\begin{array}{l}\text { Battán Horenstein et al. } \\
\text { [107-109] }\end{array}$ & $\begin{array}{l}2010,2011 \\
\quad 2012\end{array}$ & Argentina & Pigs & Insect inventory; succession; seasons; habitats \\
\hline Bonacci et al. [110] & 2010 & Italy & Pigs & Insect inventory; seasons; succession \\
\hline Carter et al. [111] & 2010 & Australia & Rats & Underground decomposition \\
\hline Chin et al. [112] & 2010 & Malaysia & Pigs & Hanging cadaver decomposition \\
\hline Cross and Simmons [113] & 2010 & UK & Pigs & Wounds \\
\hline
\end{tabular}


Table 1 (continued)

\begin{tabular}{|c|c|c|c|c|}
\hline Author(s) & $\begin{array}{l}\text { Date of } \\
\text { publication }\end{array}$ & Locality & Animal model & Major research focus \\
\hline $\begin{array}{l}\text { Matuszewski } \\
\quad \text { et al. [114-116] }\end{array}$ & 2010,2011 & Poland & Pigs & $\begin{array}{l}\text { Surface decomposition; insect inventory; seasons; } \\
\text { habitats; succession }\end{array}$ \\
\hline Michaud et al. [117] & 2010 & Canada & Pigs & Insect inventory; seasons; habitats \\
\hline Reibe and Madea [118] & 2010 & Germany & Pigs & Colonisation patterns; habitats \\
\hline Sabanoglu and Sert [119] & 2010 & Turkey & Pigs & Insect inventory; succession; seasons \\
\hline Simmons et al. [120] & 2010 & UK & Rabbits & Insect access; surface/underground decomposition \\
\hline Simmons et al. [121] & 2010 & UK & Pigs & Insect access; cadaver mass \\
\hline Swann et al. $[122,123]$ & 2010 & $\begin{array}{l}\text { Canada, } \\
\text { Australia }\end{array}$ & Pigs & Compounds released during decomposition \\
\hline Szpila et al. [124] & 2010 & $\begin{array}{l}\text { USA, } \\
\text { Poland }\end{array}$ & Pigs, rats & Colonisation of buried cadavers \\
\hline $\begin{array}{l}\text { Valdes-Perezgasga } \\
\text { et al. [125] }\end{array}$ & 2010 & Mexico & Pigs & Insect inventory; succession \\
\hline Ahmad et al. [126] & 2011 & Malaysia & Macaques & Wrapping \\
\hline Anderson [127] & 2011 & Canada & Pigs & Indoor/outdoor decomposition \\
\hline Anton et al. [128] & 2011 & Germany & Pigs & Insect inventory; succession; seasons \\
\hline Barrios and Wolff [129] & 2011 & Colombia & Pigs & $\begin{array}{l}\text { Water decomposition; arthropod inventory; } \\
\text { succession; habitats }\end{array}$ \\
\hline Bajerlein et al. [130] & 2011 & Poland & Pigs & Seasons; habitats; colonisation patterns \\
\hline Bugajski et al. [131] & 2011 & USA & Pigs & Freezing \\
\hline Cassar et al. [132] & 2011 & Australia & Pigs & Adipocere formation \\
\hline DeVault et al. [133] & 2011 & USA & Mice & Scavenging \\
\hline Dickson et al. [134] & 2011 & $\begin{array}{l}\text { New } \\
\quad \text { Zealand }\end{array}$ & Pigs (heads) & Marine decomposition; bacterial succession \\
\hline von Hoermann et al. [135] & 2011 & Germany & Pigs & Hide beetle attractants \\
\hline Spicka et al. [136] & 2011 & USA & Pigs & Cadaver mass \\
\hline Statheropoulos et al. [137] & 2011 & Greece & Pigs & Volatiles of decomposition \\
\hline Voss et al. [138] & 2011 & Australia & Pigs & Clothing \\
\hline Al-Mesbah et al. [139] & 2012 & Kuwait & Rabbits & Insect inventory; habitats; succession \\
\hline Brasseur et al. [140] & 2012 & Belgium & Pigs & Volatiles of decomposition \\
\hline Gruenthal et al. [141] & 2012 & UK & Pigs & Burnt cadaver decomposition \\
\hline Martin-Vega and Baz $[142,143]$ & 2012,2013 & Spain & Squids & Carrion and skin beetle inventory; seasons; habitats \\
\hline Ortloff et al. [144] & 2012 & Chile & Pigs & Insect inventory; succession \\
\hline Prado e Castro et al. $[145,146]$ & 2012,2013 & Portugal & Pigs & Insect inventory; succession; seasons \\
\hline Shelomi et al. [147] & 2012 & USA & Pigs & Insect repellents; blow fly colonisation patterns \\
\hline Stadler et al. [148] & 2012 & Canada & Pigs & Volatiles of decomposition \\
\hline Widya et al. [149] & 2012 & UK & Rabbits & Water decomposition; adipocere formation \\
\hline Azwandi et al. [150] & 2013 & Malaysia & $\begin{array}{l}\text { Rats, rabbits, } \\
\text { macaques }\end{array}$ & Insect inventory; succession; rat/rabbit/monkey comparison \\
\hline Barton et al. [151] & 2013 & Australia & Kangaroos & Carrion and biodiversity \\
\hline Benbow et al. [152] & 2013 & USA & Pigs & Insect inventory; succession; seasons \\
\hline Bygarski and LeBlanc [153] & 2013 & Canada & Pigs & Insect inventory; succession \\
\hline Dekeirsschieter et al. [154] & 2013 & Belgium & Pigs & Rove beetle inventory; seasons \\
\hline von Hoermann et al. [155] & 2013 & Germany & Pigs & Carrion beetle attractants \\
\hline Hyde et al. [156] & 2013 & USA & Humans & Cadaver microbiome \\
\hline Johansen et al. [157] & 2013 & Norway & Mice & Blow fly attractants \\
\hline Johnson et al. [158] & 2013 & Australia & Pigs & Thermogenesis in cadavers \\
\hline Lowe et al. [159] & 2013 & Canada & Pigs & Textiles degradation on buried cadavers \\
\hline $\begin{array}{l}\text { Matuszewski et al. [160]; } \\
\text { Mądra et al. [161] }\end{array}$ & 2013,2014 & Poland & Pigs & Insect inventory; habitats; seasons \\
\hline Metcalf et al. [162] & 2013 & USA & Mice & Cadaver microbiome \\
\hline Meyer et al. [163] & 2013 & USA & Pigs & Surface decomposition; seasons \\
\hline Sutherland et al. [164] & 2013 & $\begin{array}{l}\text { South } \\
\text { Africa }\end{array}$ & Pigs & Cadaver mass \\
\hline von der Luhe [165] & 2013 & Canada & Pigs & Compounds released into soil during decomposition \\
\hline Abouzied [166] & 2014 & $\begin{array}{l}\text { Saudi } \\
\text { Arabia }\end{array}$ & Rabbits & Insect inventory; seasons; succession \\
\hline Anderson and Bell [167] & 2014 & Canada & Pigs & Marine decomposition; arthropod inventory \\
\hline Bhadra et al. [168] & 2014 & England & Pigs (heads) & Colonisation patterns \\
\hline Caballero and León-Cortéz [169] & 2014 & Mexico & Pigs & Beetle inventory; succession; habitats \\
\hline
\end{tabular}


Table 1 (continued)

\begin{tabular}{|c|c|c|c|c|}
\hline Author(s) & $\begin{array}{l}\text { Date of } \\
\text { publication }\end{array}$ & Locality & Animal model & Major research focus \\
\hline Corrêa et al. [170] & 2014 & Brazil & Rabbits & Beetle inventory; seasons \\
\hline Farwig et al. [171] & 2014 & Germany & Mice & Biotic determinants of decomposition rate; seasons \\
\hline $\begin{array}{l}\text { Matuszewski et al. [172, 173]; } \\
\text { Mądra et al. [174] }\end{array}$ & $\begin{array}{l}2014,2016 \\
2015\end{array}$ & Poland & Pigs & $\begin{array}{l}\text { Cadaver mass; clothing; insect inventory; } \\
\text { long-term decomposition }\end{array}$ \\
\hline $\begin{array}{l}\text { Mohr and Tomberlin } \\
\quad[175,176]\end{array}$ & 2014,2015 & USA & Pigs & Cadaver visitation by adult blow flies \\
\hline Oliveira-Costa et al. [177] & 2014 & Brazil & Pigs & Succession on burnt cadavers \\
\hline Pechal et al. [178] & 2014 & USA & Pigs & Delayed insect access; colonisation patterns; succession \\
\hline Pechal et al. [179] & 2014 & USA & Pigs & Cadaver microbiome \\
\hline Perrault et al. [180-182] & 2014,2015 & Australia & Pigs & Volatiles of decomposition \\
\hline Whitaker [183] & 2014 & USA & Pigs, humans & Pig/human comparison of blow fly colonisation \\
\hline Young et al. [184] & 2014 & England & Deer & Scavenging \\
\hline Zurawski et al. [185] & 2014 & USA & Pigs & Nocturnal blow fly oviposition \\
\hline Agapiou et al. [186] & 2015 & Greece & Pigs & Volatiles of decomposition \\
\hline Alexander et al. [187] & 2015 & USA & Humans & Residual odour of decomposition in the soil \\
\hline Aubernon et al. [188] & 2015 & France & Rats & Blow fly development on contaminated cadaver \\
\hline Baz et al. [189] & 2015 & Spain & Squids & Insect inventory; habitats \\
\hline Card et al. [190] & 2015 & England & Pigs & Clothing \\
\hline Farrell et al. [191] & 2015 & Australia & Pigs & Insect inventory \\
\hline Hyde et al. [192] & 2015 & USA & Humans & Cadaver microbiome \\
\hline Iancu et al. [193] & 2015 & Romania & Pigs & Insect and microbe inventory; succession \\
\hline Iancu et al. [194] & 2015 & Romania & Pigs & Insect and microbe inventory; succession \\
\hline Lynch-Aird et al. [195] & 2015 & England & Pigs & Hanging cadaver decomposition \\
\hline Martin-Vega et al. [196] & 2015 & Spain & Squids & Clown beetle inventory; habitats \\
\hline Paczkowski et al. [197] & 2015 & Germany & Pigs & Volatiles of decomposition \\
\hline Roberts and Dabbs [198] & 2015 & USA & Pigs & Freezing \\
\hline Rysavy and Goff [199] & 2015 & Hawaii & Pigs & Underground decomposition; insect inventory \\
\hline Silahuddin et al. [200] & 2015 & Malaysia & Rabbits & Insect inventory; succession; habitats \\
\hline Stadler et al. [201] & 2015 & Canada & Pigs & Volatiles of decomposition \\
\hline Sukchit et al. [202] & 2015 & Thailand & Pigs & $\begin{array}{l}\text { Insect inventory; habitats; succession; } \\
\text { seasons; hanging; clothing }\end{array}$ \\
\hline Szpila et al. [203] & 2015 & Poland & Pigs & Insect inventory; succession \\
\hline Ueland et al. [204] & 2015 & Australia & Pigs & Textiles degradation on surface cadavers \\
\hline Zanetti et al. $[205,206]$ & 2015 & Argentina & Pigs & Underground decomposition; beetle inventory; seasons \\
\hline Zeariya et al. [207] & 2015 & Egypt & Rabbits, dogs & Insect inventory; succession; habitats \\
\hline Anderson and Bell [208] & 2016 & Canada & Pigs & Marine decomposition; seasons \\
\hline Cammack et al. [209] & 2016 & USA & Pigs & Concealment; seasons \\
\hline Lyu et al. $[210]$ & 2016 & China & Pigs & Beetle inventory \\
\hline Mashaly [211] & 2016 & Egypt & Rabbits & $\begin{array}{l}\text { Burnt cadaver decomposition; insect inventory; } \\
\text { succession; habitats }\end{array}$ \\
\hline Metcalf et al. [212] & 2016 & USA & Mice, Humans & Cadaver microbiome \\
\hline Moffatt et al. [213] & 2016 & England & Pigs & Distribution of maggots length on carrion \\
\hline Parry et al. [214] & 2016 & $\begin{array}{l}\text { South } \\
\text { Africa }\end{array}$ & Fishes & Fly inventory; habitats; seasons \\
\hline Perez et al. [215] & 2016 & USA & Pigs & Distance between cadavers \\
\hline Weidner et al. [216] & 2016 & USA & Pigs & Blow fly colonisation timing \\
\hline Weiss et al. [217] & 2016 & USA & Pigs & Cadaver microbiome \\
\hline Vasconcelos et al. [218] & 2016 & Brazil & Pigs & Fly inventory \\
\hline Amendt et al. [219] & 2017 & Germany & Pigs & Thermal imaging of cadavers \\
\hline Connor et al. [220] & 2017 & USA & Pigs, humans & Human/pig comparison \\
\hline Fancher et al. [221] & 2017 & USA & Humans & Compounds released into soil during decomposition \\
\hline Marais-Werner et al. [222] & 2017 & $\begin{array}{l}\text { South } \\
\text { Africa }\end{array}$ & Pigs & Underground decomposition \\
\hline Martin-Vega et al. [223] & 2017 & Spain & Pigs & Colonisation patterns; seasons \\
\hline Mashaly [224] & 2017 & $\begin{array}{l}\text { Saudi } \\
\text { Arabia }\end{array}$ & Rabbits & Beetle inventory; habitats; succession \\
\hline McIntosh et al. [225] & 2017 & Australia & Pigs & Burnt cadaver decomposition; succession \\
\hline Michaud and Moreau [226] & 2017 & Canada & Pigs & Succession mechanisms \\
\hline Niederegger et al. [227] & 2017 & Germany & Pigs & Wounds \\
\hline Pacheco et al. [228] & 2017 & Canada & Pigs & Blow fly colonisation patterns \\
\hline
\end{tabular}


Table 1 (continued)

\begin{tabular}{|c|c|c|c|c|}
\hline Author(s) & $\begin{array}{l}\text { Date of } \\
\text { publication }\end{array}$ & Locality & Animal model & Major research focus \\
\hline Roberts et al. [229] & 2017 & USA & Humans & Cadaver mass \\
\hline Scholl and Moffatt [230] & 2017 & England & Pigs & Dismemberment; concealment in plastic sacks \\
\hline Wang et al. [231] & 2017 & China & $\begin{array}{l}\text { Pigs, humans, } \\
\text { rabbits }\end{array}$ & $\begin{array}{l}\text { Human/pig/rabbit comparison; surface } \\
\text { decomposition; succession; }\end{array}$ \\
\hline Wang et al. [232] & 2017 & China & Pigs & Exposure daytime; succession; \\
\hline Weidner et al. [233] & 2017 & USA & Pigs & Comparison of bait traps and cadaver inventories \\
\hline Cruise et al. $[234,235]$ & 2018 & USA & Pigs & Insect inventory; succession; sampling techniques \\
\hline $\begin{array}{l}\text { Dautartas et al. [236]; } \\
\text { Steadman et al. [237] }\end{array}$ & 2018 & USA & $\begin{array}{l}\text { Pigs, humans, } \\
\text { rabbits }\end{array}$ & $\begin{array}{l}\text { Human/pig/rabbit comparison; surface } \\
\text { decomposition; scavenging }\end{array}$ \\
\hline Díaz-Aranda et al. [238] & 2018 & Spain & Pigs & Insect inventory; succession; seasons \\
\hline $\begin{array}{l}\text { Frątczak-Łagiewska and } \\
\text { Matuszewski [239] }\end{array}$ & 2018 & Poland & Pigs & Silphid beetles; succession; seasons; habitats \\
\hline von Hoermann et al. [240] & 2018 & Germany & Pigs & Carrion beetle inventory; habitats \\
\hline Knobel et al. [241] & 2018 & Australia & Pigs, humans & $\begin{array}{l}\text { Decomposition rates; odour profiles; } \\
\text { human/pig comparison }\end{array}$ \\
\hline Lee et al. [242] & 2018 & Australia & Pigs & Thermal imaging of cadavers \\
\hline Lutz et al. [243] & 2018 & Canada & Pigs & $\begin{array}{l}\text { Beetle colonisation and breeding on } \\
\text { concealed carcasses }\end{array}$ \\
\hline Mañas-Jordá et al. [244] & 2018 & Mexico & Pigs & Fly inventory; succession; habitats \\
\hline Marais-Werner et al. [245] & 2018 & $\begin{array}{l}\text { South } \\
\text { Africa }\end{array}$ & Pigs & Surface/underground decomposition \\
\hline Pérez-Marcos [246] & 2018 & Spain & Pigs, chickens & Fly inventory; pig/chicken comparison \\
\hline Salimi et al. [247] & 2018 & Iran & Rabbits & Insect inventory; succession; seasons; habitats \\
\hline Shayya [248] & 2018 & Lebanon & Pigs & Clown beetle inventory; succession; seasons; habitats \\
\hline Singh et al. [249] & 2018 & USA & Humans & $\begin{array}{l}\text { Arthropod and microbe inventory and succession } \\
\text { in the soil below a cadaver }\end{array}$ \\
\hline Spies et al. $[250,251]$ & 2018 & $\begin{array}{l}\text { South } \\
\text { Africa }\end{array}$ & Pigs & Scavenging \\
\hline Szelecz et al. $[252,253]$ & 2018 & Switzerland & Pigs & $\begin{array}{l}\text { Compounds released into soil during decomposition; clown beetle } \\
\text { colonisation of hanging and surface cadavers }\end{array}$ \\
\hline
\end{tabular}

followed the stage-based paradigm (e.g. [6]). Decay stages, named according to physiochemical changes seen in the cadaver, accompanied timetables of insect succession. Stage descriptions varied in both number and duration; moreover, the widely-held view was that the onset of each stage was marked by an abrupt change in the insect community, similar to Mégnin's [3] notion of "squads". Subsequent ecological and forensic studies found that succession in carrion largely follows a continuum of gradual changes [33-35]. Despite these findings, the use of stages of decomposition is still frequent in the forensic literature [35].

More recently, pigs became model animals in experimental research of forensic entomology and taphonomy (Table 1). Pigs have influenced recent theoretical developments in carrion and succession ecology and shaped our understanding of how vertebrate cadavers decompose in various environments, including indoor, suspended, buried, epigeic, intertidal, marine and freshwater settings. A wide spectrum of habitats has been investigated (Table 1) and found to show some idiosyncratic variations on otherwise very general patterns (Fig. 1). Results of these studies indicate that temperature and access or abundance of carrion insects are key environmental determinants of cadaver decomposition, whereas cadaver mass is a key cadaver-related determinant (Table 1, Fig. 1). At least five general decomposition patterns may be currently discerned: decay driven by either vertebrate scavengers, microbes, burying beetles, blow flies or blow flies with silphid beetles, with distinct key determinants of decomposition rate in each of the patterns (Table 1, Fig. 1).

Human cadavers vary in many characteristics that influence decomposition, most of which have been investigated using pigs (Table 1). Pre- or postmortem modifications such as wounds, burning, wrapping, dismemberment, contamination, concealment and clothing may affect the colonisation process and eventually decomposition to varying degrees, depending on their intensity and context of action (Table 1). Some modifications do not affect the whole cadaver, leaving parts of it to be colonized by insects in their usual manner, while other modifications such as clothing have effects on insect colonisation or succession that are too small or too variable to have practical consequences for estimates of postmortem intervals (PMIs). Other modifications delay colonisation by insects but have little consequence once colonisation has occurred. The same modifications may however 


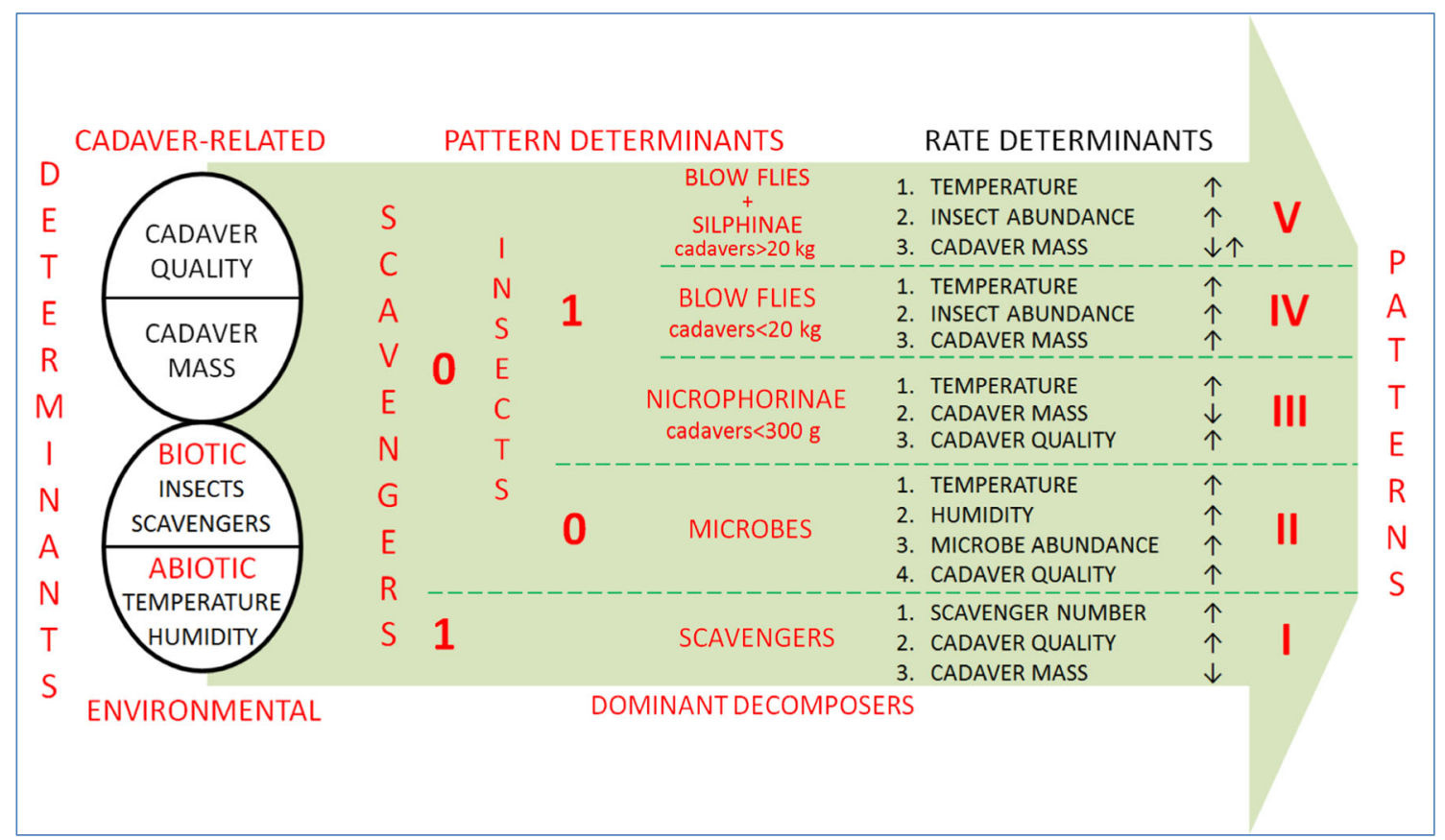

Fig. 1 Determinants and general patterns of cadaver decompositionsynthesis based on findings of cadaver decomposition studies (Table 1). Numbers $\mathrm{I}-\mathrm{V}$ denote general patterns of decomposition (differing according to dominant decomposers, key determinants of decomposition rate and the effect they have on decomposition). Numbers " 0 " and " 1 " denote absence and presence of scavengers or insects. Arrows next to rate determinants indicate whether a determinant, considered in isolation, is positively $(\uparrow)$ or negatively $(\downarrow)$ related to decomposition rate. Some determinants in this figure should be considered as sets of simple determinants, e.g. cadaver quality including body mass index, antemortem cadaver modifications (e.g. pharmaceuticals use), postmortem modifications (e.g. freezing during the winter) and others differently affect non-entomological processes, for example, clothing influences rate of cadaver cooling and therefore is considered important for some pathology-based methods for estimation of PMI, e.g. Henssge's nomogram method [36]. Regarding insects, the implications of modification appear to be larger than the effect of the cadaver's species.

In parallel, pig cadavers were used to test new forensic techniques or validate well-established ones (Table 2). They have provided proof-of-concept for techniques as simple as entomological sampling and as sophisticated as ground penetrating radar or thermal imaging to locate cadavers (Table 2). Many of these techniques have gone on to be applied to forensic investigations involving humans, demonstrating in this way the practicality of pigs as model cadavers.

\section{Are pigs an appropriate model for forensic entomology and taphonomy?}

A comparison of the advantages and disadvantages of pig and human cadavers for experimental forensic entomology and taphonomy research (Table 3) indicates that pigs are usually superior to humans in such experiments. Most importantly, pig cadavers may easily be replicated in large numbers and at low cost, whereas access to human corpses is restricted to taphonomic facilities or medical examiner's offices with all of their associated inherent difficulties. At taphonomic facilities, waiting times for receiving replicate bodies on multipledonation days are unpredictable and uncontrollable [37], even if minimum criteria are met for accepting cadavers as "replicates" (i.e. death within $48 \mathrm{~h}$ of acquisition, intact, unautopsied, unembalmed and refrigerated). The difficulty in amassing replicate human cadavers allows little experimental control over key decomposition determinants such as cadaver mass. The unpredictable and uncontrollable variation inherent in cadaver availability may limit the value of observations in humans and invalidate the experiment, by producing statistically underpowered comparisons that are insufficient to detect significant differences and by enlarging the risk of confounding effects. In addition, the practical realities of working with human remains can limit the types of information that can be gleaned from and about them. Moreover, the continual association of the taphonomy facilities with human cadavers can itself present a challenge. Although a 1998 field study at the Tennessee facility found little evidence of cadaver enrichment effects on the surface-active entomofauna or decay rates using pig carcasses [38, 39], a recent study of soil parameters [40] demonstrated that the Tennessee site is contaminated with high levels of decomposition products, which may limit the 
Table 2 Forensic methods and techniques developed, refined or tested using pig cadavers. References to this table are listed in Electronic Supplementary Material

\begin{tabular}{|c|c|c|}
\hline Method/technique & References & Pig cadaver use \\
\hline $\begin{array}{l}\text { Field protocol for experimental } \\
\text { studies on PMI }\end{array}$ & Schoenly et al. $[1,2]$ & Tests of the protocol \\
\hline \multirow[t]{2}{*}{ Model organisms } & Watson and Carlton $[3,4]$ & Comparisons of different animals \\
\hline & $\begin{array}{l}\text { Schoenly et al. [2]; Wang et al. [5]; } \\
\text { Connor et al. [6]; Dautartas et al. [7] }\end{array}$ & Comparisons of pigs and humans \\
\hline $\begin{array}{l}\text { Human-size insect trap for studying } \\
\text { succession }\end{array}$ & Schoenly et al. [1] & $\begin{array}{l}\text { Recorded trap microclimate and carrion-arthropod } \\
\text { families caught by trap }\end{array}$ \\
\hline $\begin{array}{l}\text { Device for sampling cadaver-related } \\
\text { aquatic insects }\end{array}$ & Vance et al. [8] & Tests of trap efficiency in catching aquatic insects \\
\hline $\begin{array}{l}\text { Degree-day index for decomposition } \\
\text { related processes }\end{array}$ & Michaud and Moreau [9] & Development of the index and tests for its reliability \\
\hline Reconstruction of temperature conditions & Hofer et al. [10] & Reliability of temperature recordings on a death scene \\
\hline \multirow{4}{*}{$\begin{array}{l}\text { Temperature methods for insect } \\
\text { pre-appearance interval (PAI) }\end{array}$} & Matuszewski $[11,12]$ & Development of PAI models; tests of the method \\
\hline & $\begin{array}{l}\text { Matuszewski and Szafałowicz [13]; Archer [14]; } \\
\text { Matuszewski et al. [15] }\end{array}$ & Development of PAI models \\
\hline & Matuszewski and Mądra 2015 [16] & Tests of the protocols for PAI field studies \\
\hline & Matuszewski and Mądra-Bielewicz [17] & Validation of PAI methods \\
\hline \multirow[t]{5}{*}{ Total body score } & Myburgh et al. [18] & Validation of the method \\
\hline & Lynch-Aird et al. [19] & Development of TBS for hanging cadavers \\
\hline & Nawrocka et al. [20] & Inter-rater reliability of the TBS \\
\hline & Keough et al. [21] & Amendment of TBS for pig cadavers \\
\hline & Ribéreau-Gayon et al. [22] & Reliability of TBS based on cadaver pictures \\
\hline \multirow[t]{8}{*}{ PMI estimation based on insect succession } & Michaud and Moreau [23] & $\begin{array}{l}\text { Tests of predictability of insect occurrence based on degree-day } \\
\text { accumulation }\end{array}$ \\
\hline & Michaud and Moreau [24] & Tests of sampling protocols for field studies \\
\hline & Perez et al. [25] & $\begin{array}{l}\text { Evaluation of utility of insect taxa for derivation of confidence } \\
\text { intervals about PMI estimate }\end{array}$ \\
\hline & Mohr and Tomberlin [26] & $\begin{array}{l}\text { Tests of oocyte development of adult blow flies visiting cadaver } \\
\text { as a PMI indicator }\end{array}$ \\
\hline & Perez et al. [27] & $\begin{array}{l}\text { Tests of minimum inter-cadaver distances for forensic field } \\
\text { studies }\end{array}$ \\
\hline & Matuszewski [28] & $\begin{array}{l}\text { Tests of presence/absence of insect taxa as an approach for PMI } \\
\text { estimation }\end{array}$ \\
\hline & Mądra-Bielewicz et al. [29] & Tests of insect sex and size as PMI indicators \\
\hline & Cruise et al. $[30]$ & Tests of the protocols for cadaver field studies \\
\hline \multirow{3}{*}{$\begin{array}{l}\text { PMI estimation based on insect } \\
\text { development }\end{array}$} & VanLaerhoven [31] & Validation of methods \\
\hline & Reibe-Pal and Madea [32] & Comparison of methods \\
\hline & Weatherbee et al. [33] & Validation of methods \\
\hline PMI estimation based on microbes & Pechal et al. [34] & Tests of usefulness of microbe succession for PMI estimation \\
\hline Exposed cadavers searching & Amendt et al. [35]; Lee et al. [36] & Tests of thermal imaging techniques used from the air \\
\hline Clandestine burial searching & $\begin{array}{l}\text { Schultz et al. [37]; Schultz [38]; } \\
\text { Salsarola et al. [39] }\end{array}$ & Tests of ground-penetrating radar \\
\hline Submerged cadavers searching & Healy et al. [40] & Tests of side-scan sonar \\
\hline Detection of gasoline in cadaver tissues & Pahor et al. [41] & Proof-of-concept tests \\
\hline
\end{tabular}

interpretation of certain nutrient-based taphonomic results as no reliable baseline sample can be obtained within the facility.

While, in many cases, researchers may be interested in how the decomposition process works in humans, the available human remains are either derived from inappropriate populations, cannot be linked to control samples or are too variable for robust experiments. Due to these practicalities, pig cadavers are usually the best choice available for most experimental purposes in forensic sciences. Moreover, pig cadavers may be used to compare treatments of relevance with forensic scenarios and to make inferences about human decomposition. If treatment $\mathrm{A}$ results in a slower decomposition than treatment B in pigs, in the absence of other information, we can reasonably assume a similar effect in humans, especially if it can be supported with other knowledge and logic. The possibility that a model animal and the humans that it models decompose differently does not make that model useless; it depends on the specific question being addressed. This conclusion has much wider applicability. For example, mouse cadavers were useful in demonstrating forensic applications of microbiology $[41,42]$. Postmortem microbiome comparisons between different animals revealed the common appearance of some informative bacterial taxa across rodent, pig 
and human models [41-43]. Another example is the use of rabbit cadavers to provide local carrion insect inventories (Table 1). When early cadaver colonizers (e.g. blow flies) are the focus, rabbits are as informative as pigs or humans, but when middle or late colonizers (e.g. beetles of Silphinae or Cleridae) are studied, rabbit cadavers are inappropriate, because such insects rarely colonize carcasses as small as rabbits $[44,45]$.

Comparative studies of pig and human cadavers revealed largely overlapping insect faunas [14, 44], with as much difference between individual pigs or humans as between pigs and humans [46]. Similarly, insect faunas compiled from human case studies (e.g. $[47,48]$ ) largely resembled those from pig cadaver experiments (Table 1). Although alligator carrion revealed important faunal differences compared with large mammals (i.e. pigs, bears and deer), the latter group yielded highly similar insect community composition $[49,50]$. These results indicate that, when compared across related cadaver taxa of similar size, carrion insects (i.e. necrophagous insects) show negligible preference for one cadaver taxon over another. Therefore, when pig cadavers are used to inventory local carrion-arthropod faunas, they appear to be as good as humans and are more practical (Table 3).

However, we suggest that pig cadavers larger than the recommended 20-30 kg domestic pigs $[9,10]$ should be used to compile full inventories of carrion entomofauna because smaller pigs yield an incomplete insect inventory (i.e. underrepresentation of middle or late colonizers $[44,45])$. We therefore recommend cadavers a starting mass of at least $40 \mathrm{~kg}$ (and preferably $50-80 \mathrm{~kg}$ ) as a standard to investigate local carrioninsect inventories. Smaller cadavers (piglets or rabbits) may be used in cases when early colonizers (e.g. blow flies) are the focus.

Most methods developed in forensic entomology or taphonomy are intended to be used with human cadavers. Therefore, at least their final validation should be performed with humans and preferably in real case scenarios. We are not aware, however, of any validation experiment in which performance of the forensic method developed using non-human cadavers has been evaluated using human cadavers. This is definitely an area for future experiments. Such research could enable forensic scientists to evaluate whether techniques based on data from human analogues (e.g. pig cadavers) are satisfactorily accurate when used in casework for human cadavers. As a result, we could distinguish techniques for which reference data could be amassed using human cadaver analogues and techniques for which human cadavers are necessary to get reference data. Nevertheless, analogues for humans, particularly large-bodied species, serve well in "proof-ofconcept" studies (Table 2). Similarly, initial validation of forensic methods may be efficiently performed with pig cadavers (Table 2), particularly when different cadaver traits (e.g. mass) or environmental conditions (e.g. below/ above ground) are to be compared.

All animals used in forensic entomology or taphonomy research are highly variable within species. This may lead to misinterpretation of experimental results, particularly when the experimental design of a study has weaknesses (see section 4 of this paper). However, the variation may also be advantageous, as it enables the researcher to choose the model best suited to the research. For example, if the scientific question obliges large replication, the experiment simply cannot be made with large pigs within standard research budgets, whereas piglets may be appropriate. If the researcher is interested in the thermal profile of decomposing remains, it may be more important to focus on the sunlight absorbance and mass of the model species than on its other traits. This argument may be extended to different animal models: experiments on initial colonisation patterns of blow flies may be more tractable using piglets or rabbits rather than adult pig or human cadavers. On the other hand, validation of the total body score (TBS) method for PMI estimation [51] needs humans or at least large pigs. Therefore, there is no universal model cadaver for research in forensic taphonomy or entomology, and the one that should be chosen depends on the scientific question and its experimental demands. This is an important point for the forensic science community to consider when designing experiments, analysing results or extrapolating conclusions.

\section{Critique of the pig model as an analogue for human cadavers}

\section{Background}

Use of domestic pigs in experimental forensic sciences has been challenged by recent comparisons of pig and human cadaver decomposition [23, 24]. One study [23] concluded that "pigs are not an adequate proxy for human decomposition studies", and another [24] indicated that neither rabbits nor pigs "captured the pattern, rate, and variability of human decomposition". Pigs may indeed decompose differently to humans, and therefore their experimental comparison is clearly worthwhile to forensic sciences. However, the intrinsic logistical difficulties associated with experiments involving human cadavers may impair such comparisons (Table 3), and therefore, questions arise about the validity of recent findings and conclusions. In the following sections, we discuss these questions and try to identify their consequences for the findings of the referenced experiments $[23,24]$ and the implications they have for the validity of the conclusion that pigs are inadequate analogues for humans in forensic research. 
Table 3 Advantages and disadvantages of domestic pig and human cadavers in forensic entomology and taphonomy research related to human decomposition $[6,9-11,14,23$, $24,44,65]$

\begin{tabular}{|c|c|c|}
\hline & Pig cadavers & Human cadavers \\
\hline Cons & $\begin{array}{l}\text { 1. Dissimilar to human cadavers in some } \\
\text { important aspects: } \\
\text { a. Body proportions } \\
\text { b. Gastrointestinal anatomy } \\
\text { c. Diet (more uniform, larger proportion of plant } \\
\text { products) } \\
\text { 2. More uniform than humans } \\
\text { 3. Unacceptable in some cultures }\end{array}$ & $\begin{array}{l}\text { 1. Difficult to replicate: } \\
\text { a. Available in low numbers } \\
\text { b. Time and cause of death beyond researcher control } \\
\text { (self-donation, age, disease incidence etc.) } \\
\text { c. Dissimilar to each other in: } \\
\text { - Mass } \\
\text { - Age, sex, ethnicity } \\
\text { - Antemortem pharmaceuticals use } \\
\text { - Body conditions (frozen/fresh, } \\
\text { autopsied/non-autopsied, etc.) } \\
\text { 2. Limitations of taphonomy facilities (body farms): } \\
\text { a. Small area, potential for insufficient inter-cadaver } \\
\text { distances } \\
\text { b. Uniform abiotic conditions } \\
\text { c. Frequently non-natural conditions } \\
\text { d. Area saturated with cadavers } \\
\text { 3. Limitations of casework (i.e. medical examiner } \\
\text { samples): } \\
\text { a. Restricted to observation } \\
\text { b. Cannot control effects of routine processing of remains } \\
\text { c. Sometimes no information about death circumstances } \\
\text { and the cadaver itself } \\
\text { 4. Risk of sensationalized research } \\
\text { a. Complex ethical considerations/generally } \\
\text { unacceptable } \\
\text { b. Potentially negative publicity }\end{array}$ \\
\hline Pros & $\begin{array}{l}\text { 1. Similar to human cadavers in some important } \\
\text { aspects: } \\
\text { a. Body mass range } \\
\text { b. Anatomy } \\
\text { c. Body composition } \\
\text { d. Skin coverage with hair } \\
\text { e. Gut microbiota } \\
\text { f. Gross processes of decay } \\
\text { 2. Easy to replicate: } \\
\text { a. Cheap and available in large numbers } \\
\text { b. Time and cause of death controllable } \\
\text { c. Cadaver traits controllable } \\
\text { d. Possible to work with unfrozen cadavers } \\
\text { 3. Less sensationalized research and relatively } \\
\text { straightforward ethical considerations }\end{array}$ & $\begin{array}{l}\text { c. Potential for findings to be "oversold" } \\
\text { 1. No species-related differences }\end{array}$ \\
\hline
\end{tabular}

As we have discussed in section 3, all model organisms are highly variable intra-specifically. Biased sampling of this variation may lead to the misinterpretation of results of any model comparison. Both pigs and humans clearly exhibit variable sizes, pigmentation, hairiness, body mass index and other characteristics. Such factors may be confounded with treatments, and when they affect decomposition, they may make it impossible to assign results of a comparison between human and pig cadavers (or any other model) to a species effect (Fig. 2). As an example, if a study was conducted with male piglets and adult female humans only, it would not be possible to disentangle sex and age (or mass) effects from species effects. Therefore, sample selection within and between species is critical for such comparisons. 


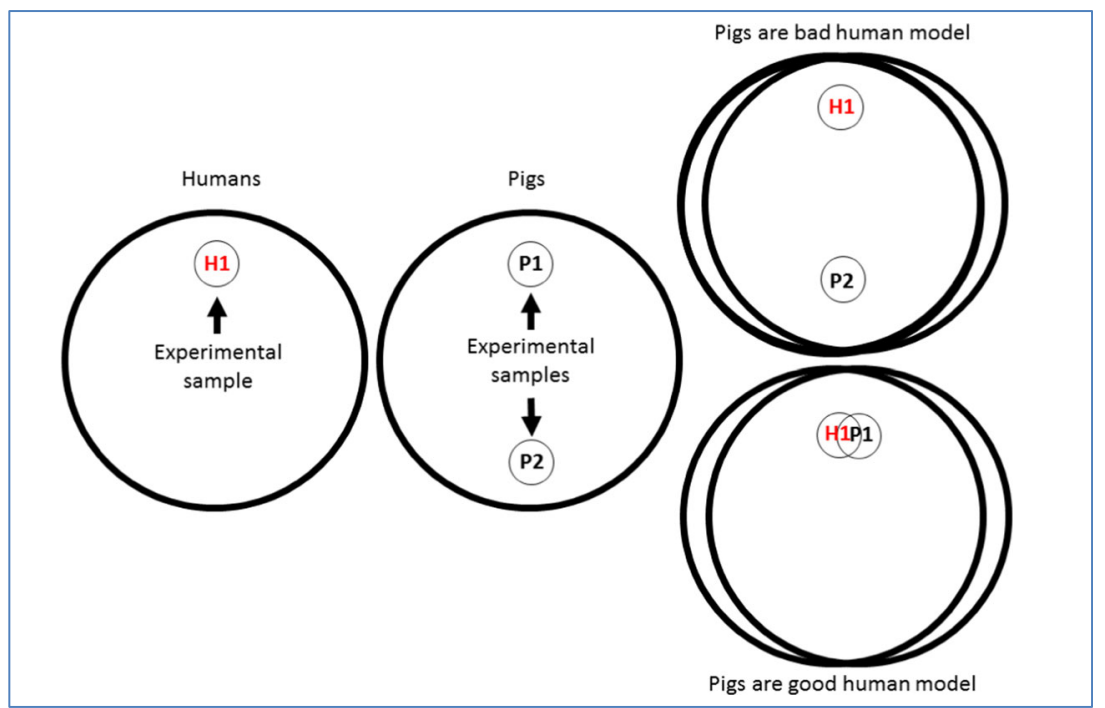

Fig. 2 Schematic representation of dangers for human/pig comparisons, resulting from intraspecific variation of pigs and humans. Large circles are phenotype spaces (for a species), small circles inside are experimental samples of pigs or humans. The samples can come from anywhere within the phenotype space for the species, but if comparisons are to be made between species, it is desirable that the samples come from the phenotype

\section{Experimental design}

\section{Confounded variables}

Confounded variables make the outcome of an experiment ambiguous. Confounding effects arise when differences recorded in a response (dependent) variable as a putative result of experimental manipulation of explanatory (independent) variable(s) cannot be separated from other variables that may affect the response [52]. To confidently show that differences resulted from experimental manipulations, the groups under comparison should differ only in the manipulated variable(s), or more realistically, the groups should not differ systematically in any space shared by both species. Thus, it is possible to design an experiment comparing the same two species and either properly (bottom circles, e.g. large humans versus large pigs) or improperly (upper circles, e.g. large humans versus small pigs) compare the species, depending on the choice or availability of sampled individuals

important variable other than the one under manipulation. Confounding variables should be controlled in the experimental design (and thus eliminated) or in its statistical analysis (and thus quantified). An important confounding variable likely to arise in pig and human comparisons is body mass.

Identifying differences in decomposition between species needs an experiment in which cadaver samples differ systematically only in the cadavers' species. In the experiments of Dautartas et al. [24] and Connor et al. [23], samples of pig and human cadavers differed systematically in cadaver mass: the humans were systematically much larger than the pigs (Table 4). Although there are anecdotal observations suggesting low importance of adult human cadaver mass [53] and

Table 4 Cadaver mass of pigs and humans used by Dautartas et al. [24] and Connor et al. [23]

Cadaver mass $(\mathrm{kg})$

\begin{tabular}{|c|c|c|c|c|c|c|c|}
\hline \multicolumn{3}{|l|}{ Pigs } & \multirow{2}{*}{$\begin{array}{l}\text { Difference between humans } \\
\text { and pigs in mean cadaver mass }\end{array}$} & \multicolumn{3}{|l|}{ Humans } & \multirow[b]{2}{*}{$\begin{array}{l}\text { Dissimilarity score } \\
(\mathrm{h}-\mathrm{p}) /(\mathrm{h}+\mathrm{p})\end{array}$} \\
\hline Mean & Range & $\mathrm{V}$ & & Mean & Range & $\mathrm{V}$ & \\
\hline 64.6 & $60-68$ & 4.8 & 13.2 & 77.8 & $72-84$ & 6.1 & 0.093 \\
\hline 49 & $40-59$ & 14.1 & 25 & 74 & 53-107 & 30.8 & 0.203 \\
\hline 50.6 & $47-57$ & 8.5 & 24.8 & 75.4 & $57-85$ & 15.1 & 0.197 \\
\hline 35 (median) & $25-64$ & $\mathrm{n} / \mathrm{a}$ & $\geq 45^{*}($ median $)$ & $\mathrm{n} / \mathrm{a}(\geq 80)^{*}$ & $\mathrm{n} / \mathrm{a}$ & $\mathrm{n} / \mathrm{a}$ & $0.391 *$ \\
\hline
\end{tabular}

n/a not available

*Authors did not report mass of their human cadavers. They used adult humans and mention that "...over half the human sample was overweight or obese.". According to "Anthropometric Reference Data for Children and Adults: United States, 2011-2014" [Fryar et al., 2016, Vital Health Stat 3] average body weight of adult females in USA was $76.4 \mathrm{~kg}$ and adult males $88.8 \mathrm{~kg}$. Based on these data, we assume that the median mass of the human sample from Connor et al. [23] was no less than $80 \mathrm{~kg}$, so the difference in median between pig and human sample was no less than $45 \mathrm{~kg}$ 
experimental findings supporting the claim that in a mass range of $73-159 \mathrm{~kg}(N=12$, nine cadavers over $100 \mathrm{~kg}$, i.e. obese, adipose bodies) decomposition rate is not significantly related to human body mass [54], all rigorous studies revealed that in a forensically relevant mass range $(10-90 \mathrm{~kg})$ small pig cadavers decompose significantly faster than large ones [55-59]. This difference appeared only in the case of insect-colonized carcasses [56] and has been suggested to result from less efficient active decay in larger cadavers, as a consequence of competition over carrion between different insect taxa [45, 59]. It is also related to surface-tovolume ratios, which reflect the surface area of the tissue where insects can feed, and to the size of the individual insect relative to that of the resource. Based on these patterns, it may be assumed that, when insects are present,

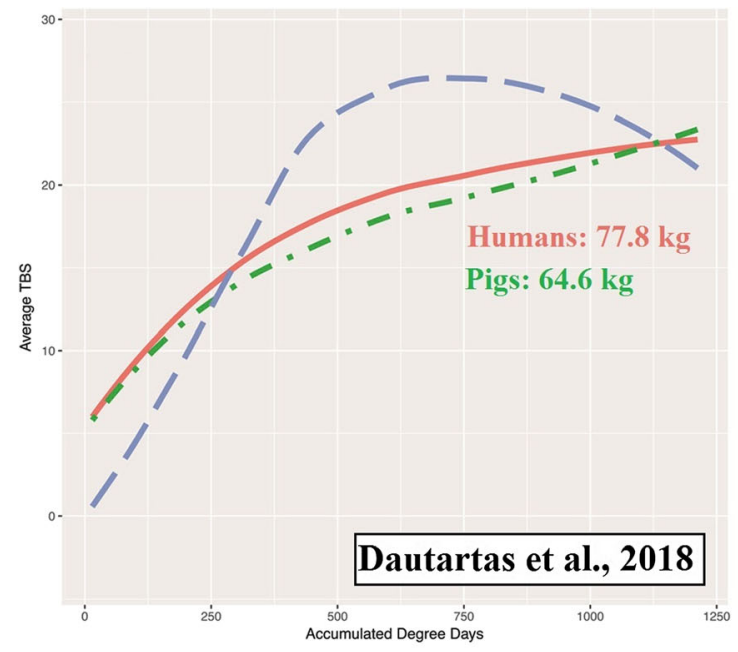

smaller pig cadavers' progress through the TBS scale at a faster rate than larger human cadavers. This seems to be the case (Figs. 3 and 4) with the studies of Dautartas et al. [24] and Connor et al. [23], making some of their results ambiguous and uninterpretable with respect to human-pig differences.

\section{Independence of replicates. Distance between cadavers}

When cadavers are close to one other, they may crosscontaminate one another or "compete" for insect colonizers, or both, making them statistically non-independent $[60,61]$. The cadaver that is more attractive to insects may mask the other, resulting in underrepresentation of insects and slower decomposition of the less attractive cadaver. In addition,

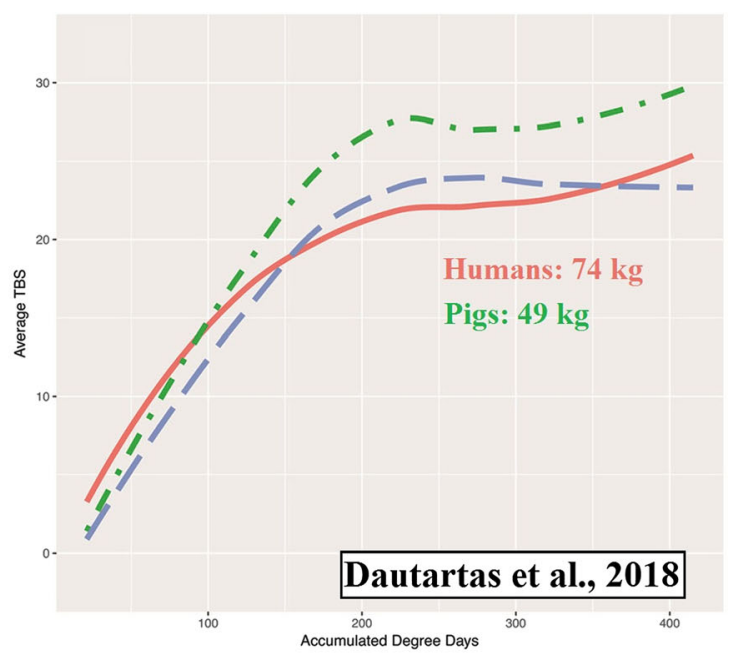

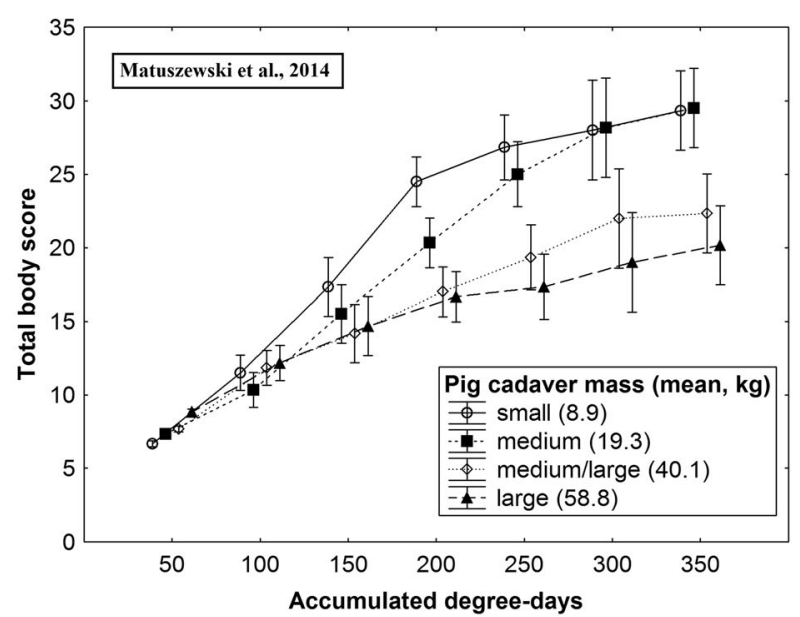

Fig. 3 Changes in total body score (TBS) during decomposition of pig and human cadavers. Upper panel shows Fig. 2A and 2B from Dautartas et al. [24] displaying results of their trial 1 (Fig. 2A, spring, insects present) and trial 2 (figure $2 \mathrm{~B}$, summer, insects present). Lower figure is a modification of Fig. 13 from Matuszewski et al. [59], displaying results of their experiment with pig cadavers of different mass. Red lines in Dautartas et al. [24] are for human cadavers, green lines for pig cadavers. Comparison of the trials 1 and 2 (upper panel) indicates that an increase of difference in cadaver mass between pigs and humans in the trial 2 was followed by larger difference between TBS curves. Moreover, differences between TBS curves in the trial 2 are similar to differences between medium/large and large pig cadavers in the experiment of Matuszewski et al. [59]. Therefore, the differences between pigs and humans in Fig. 2B of Dautartas et al. [24] may be interpreted as the result of differences in mass between the cadavers and not differences in the species of cadaver 


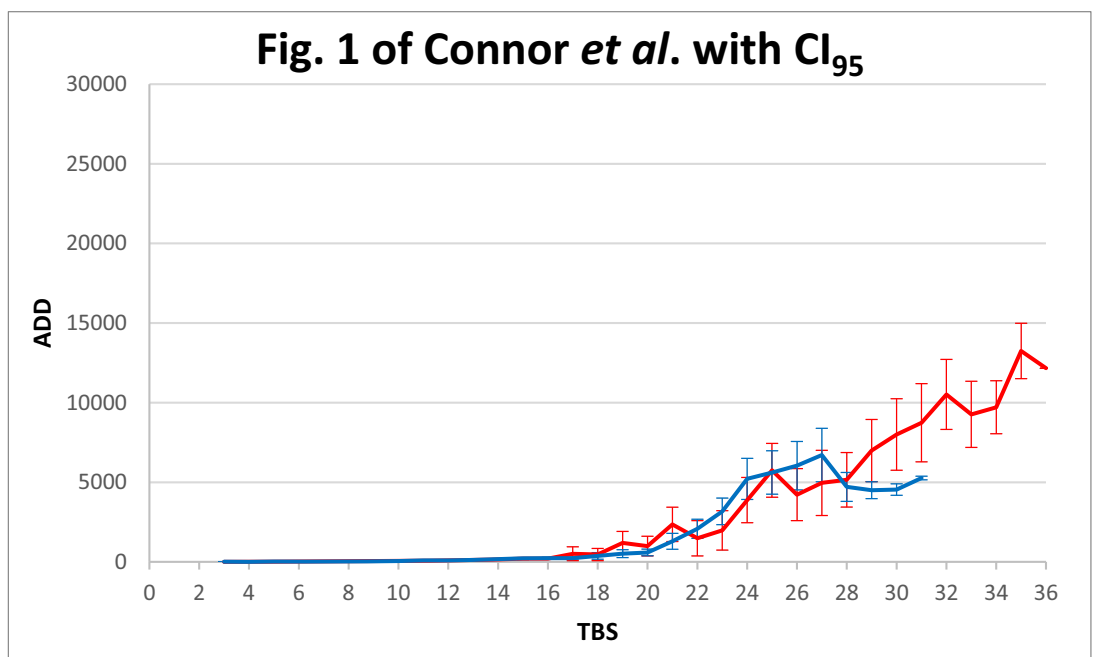

Fig. 4 Total body score (TBS) and accumulated degree-days (ADD) with 95\% confidence intervals for ADD added and plotted based on data from Table 1 of Connor et al., [23], presented by these authors (as Fig. 1) without confidence intervals. The $95 \%$ confidence intervals presented

dispersal of larvae becomes a potential mechanism to affect larval competition if the carcasses are located close to one another. If such effects are not taken into account (i.e., watching for larval dispersal, deploying drift fencing), small inter-cadaver distances are likely to alter species composition or decomposition rate, and lead to a lack of independence of experimental units, a basic assumption or requirement of most statistical tests.

In forensic entomology experiments, cadavers have usually been placed at least $50 \mathrm{~m}$ apart (Table 1) because there is empirical support that such a distance is sufficient to minimize cross-contamination by dispersing fly larvae $[62,63]$ and to ensure independence of cadavers [60]. In forensic taphonomy experiments, particularly with human cadavers, the distance has usually been much smaller, probably as a result of the smaller areas of human taphonomy research facilities where such experiments are located. Dautartas et al. [24] report that their cadavers were placed at least $3 \mathrm{~m}$ apart, and although Connor et al. [23] provide no information on the distance between their cadavers, the outdoor facility where the study was located has an area of about one acre [22], so we can assume their between-cadaver distances were less than $50 \mathrm{~m}$. Such distances indicate that the cadavers used in both studies were not demonstrably independent in terms of the insect communities attending them. Little is known about the effect of small distance between cadavers on the pattern and rate of insect-mediated decomposition $[60,61]$; therefore, relevant consequences of small between-cadaver distance on the results of the above studies are currently difficult to identify.

\section{Inter-annual effects}

Different years generally have different weather profiles leading to different insect richness and abundances and/or in this figure used standard deviations calculated from coefficients of variation reported in Table 1 of Connor et al. [23]. Red lines-pig cadavers; blue lines - human cadavers

different insect pre-appearance intervals (PAI) (Table 1). These may result in substantial annual differences in decomposition rate.

In the experiments of Connor et al. [23], pig cadavers were exposed in September 2012 through August 2013 (12 pigs, one each month), while an extra five pigs were exposed on the same day as their 2nd through 6th human cadaver. The authors gave no specific dates of the human cadaver exposure (between September 2012 and December 2015). However, according to Wikipedia [22], they started to use human cadavers at their outdoor facility in November 2013. Therefore, most pigs were exposed in 2012 and 2013 and most humans probably in 2014 and 2015. If that was the case, there was a high level of treatment segregation and the species effect was confounded with an inter-annual effect. Consequently, the findings reported by Connor et al. [23] may be the result, at least in part, of differences in the biotic and abiotic determinants of decomposition in the different years of exposure rather than differences between cadaver species.

\section{Subject variables}

Subject variables are characteristics of individuals that are idiosyncratic and may affect the research variables, primarily by increasing their measured variances, sometimes referred to as "statistical noise". Wherever possible, such variables should be controlled by selecting experimental subjects to minimize their effects, usually through matching the individuals as closely as possible. This is generally possible with pigs or rabbits but can be impractical with humans. For instance, the study of Connor et al. [23] exposed some human cadavers effectively fresh at the day of death but others after 53 days of postmortem refrigeration. Refrigeration affects bacterial 
communities that initiate decomposition, with consequences for the rate of decomposition and the attraction of insects [64], which must have resulted in amplifying variation in decomposition rates of humans in that study. This sort of consequence of working with human cadavers may predispose a study to generate misleading results.

\section{Quantifying decomposition}

The total body score (TBS) was originally developed as a pointbased, semi-quantitative scale for scoring the decomposition of human cadavers [51]. It represents the total amount of accumulated decomposition identified from three body regions (head and neck, trunk, and limbs). The scale was modified for rabbit [25] and pig cadavers [65]. Keough et al. [65] observed significant differences between pig and human cadavers during early decomposition and proposed the amendment of the TBS scale for pig cadavers. The use of the same TBS scale to compare human and pig decomposition rate (e.g. [23, 24]) is incorrect. Given the differences observed between human and pig cadavers in gross morphological changes during decomposition $[23,24,65]$, cross-species use of the same TBS scale is risky and should, ideally, be complemented with other measures of decomposition, such as daily or periodic weight loss (in \%).

\section{Statistical analysis and the presentation of results}

Criticism is essential to the advancement of science but for a critique to be acceptable, its analysis must be robust. However, the analyses presented in Connor et al. [23] and Dautartas et al. [24] are inadequate to support their conclusions. In Connor et al. [23], the conclusion of a difference between human and pig cadavers is derived from a comparison of the slopes developed using linear mixed modelling. However, a simple look at the regression lines used to compare decomposition rates (see Fig. 4 in Connor et al. [23]) shows that the selected models are inadequate in terms of adjustment, leverage values and residuals. The figure also demonstrates that a statistical difference is found by the authors only because pigs were allowed to decompose for a longer period, as no human cadaver was scored at TBS values $>31$. TBS values $>31$ had a powerful leverage effect on the regression line because these scores were squared in the analysis. The analyses of Dautartas et al. [24] are also problematic because none of them accounts for repeated measurements on cadavers, resulting in temporal pseudoreplication, which is known to artificially decrease $P$ values.

In addition, statistically detectable effects may be too small or too variable to have practical significance for estimates of PMIs [66]. Because cadavers are highly variable, not surprisingly, decomposition rates can be highly variable too. For this reason, when trends are reported, they should be accompanied by quantitative indications of variation (i.e. uncertainty). For instance, human and pig cadavers appeared to decompose differently in the study of Connor et al. [23], but when 95\% confidence intervals are added to the trend lines (Fig. 4), the apparent differences disappear. The inclusion of those intervals would indicate that pigs of small size are adequate models for human decomposition unless the TBS is greater than 28 , which is a different interpretation from the one originally drawn from that research.

\section{Alternative model organisms}

In some countries, pigs are not a realistic option for religious reasons, and other animal models are needed.

Table 5 Guidelines for cadaver choice in forensic science research

\begin{tabular}{|c|c|c|c|}
\hline \multirow[t]{2}{*}{ Research type/subtype } & & \multicolumn{2}{|l|}{ Guidelines } \\
\hline & & Cadaver species & Cadaver mass \\
\hline Experimental studies & & $\begin{array}{l}\text { Domestic pig, rabbit or rodents, depending } \\
\text { on the objective of the study, human for model } \\
\text { comparison experiments }\end{array}$ & Depending on study objective \\
\hline \multirow{3}{*}{$\begin{array}{l}\text { Local insect inventory or } \\
\text { succession studies, insect } \\
\text { PAI studies }\end{array}$} & Early colonizers & Domestic pig, rabbit & No cadaver mass limitations \\
\hline & $\begin{array}{l}\text { Early and middle } \\
\text { colonizers }\end{array}$ & Domestic pig & $\begin{array}{l}\geq 20 \mathrm{~kg} \text { starting mass, preferably } \\
20-40 \mathrm{~kg}\end{array}$ \\
\hline & All colonizers & Domestic pig & $\begin{array}{l}\geq 40 \mathrm{~kg} \text { starting mass, preferably } \\
50-80 \mathrm{~kg}\end{array}$ \\
\hline \multirow[t]{3}{*}{ Tests of forensic methods } & $\begin{array}{l}\text { Proof-of-concept } \\
\text { studies }\end{array}$ & $\begin{array}{l}\text { Domestic pig, rabbit or rodents, } \\
\text { depending on method tested }\end{array}$ & Depending on method tested \\
\hline & $\begin{array}{l}\text { Initial validation } \\
\text { studies }\end{array}$ & Domestic pig & $\begin{array}{c}10-40 \mathrm{~kg} \text { as juvenile analogues, } \\
50-80 \mathrm{~kg} \text { as adult analogues }\end{array}$ \\
\hline & $\begin{array}{l}\text { Final validation } \\
\text { studies }\end{array}$ & Human & Preferably whole mass range \\
\hline
\end{tabular}


Rabbits have been frequently used by forensic entomologists (Table 1), but obviously, they are too small to serve well for most forensic research. Carrion insect assemblages are distinctly less complex and persist for less time on small-sized cadavers compared with larger cadavers $[44,45]$. Owing to their small size, the decomposition rate of rabbit cadavers is much faster than that of pig or human cadavers [24, 44]. Accordingly, the well-established importance of body size needs to be remembered when selecting alternatives, like sheep or goats, usually shorn to make insect sampling feasible and to reduce the potential impact of the fleece on decomposition, which is different from pig and human situations.

\section{Recommendations}

Previous papers suggested that a universal model cadaver for experimental field studies and training programs in forensic entomology would be a domestic pig weighing $20-30 \mathrm{~kg}$ of starting mass $[9,10]$. No recommendation is currently available for taphonomy studies. However, a single and universal "model cadaver" for the forensic sciences is not useful. Different studies have different purposes, conditions and limitations. Therefore, more flexible guidelines on cadaver species and mass are needed (Table 5). A review of the guidelines proposed in this paper (Table 5) indicates that human cadavers appear necessary only in comparative studies involving other cadaver taxa and for final validation of forensic methods. In most cases, pig cadavers are an ideal choice, whereas other animal cadavers may be useful in supplemental or unavoidable (substitutional) cases. Moreover, researchers should usually use cadavers that are larger than the currently recommended size of $20-30 \mathrm{~kg}$. Depending on the specific question of interest, other non-mass-related considerations may also be necessary.

\section{Conclusions}

Pig cadavers have provided a comprehensive experimental foundation for empirical studies of decomposition in forensic entomology, taphonomy and ecology, and are likely to remain the analogue of choice in most such studies for the immediate future. A pivotal limitation to the value of human cadavers is an adequate supply of donated bodies, especially when a well-replicated experiment is required. Some of these limitations can be avoided by conducting observational studies with samples derived from death investigations (i.e. through collaboration with medical examiners), which will be limited by the samples available, and may not be appropriate for all types of scientific questions. Analogue models such as pigs are likely to remain logistically more tractable, being more readily available, more uniform in size and age and less ethically complex to deploy. Pigs are a sensible compromise between availability, cost, ethics and similarity to humans, and there is no better candidate at this time. At present, experiments using analogues are easier to replicate and make control of confounding factors more practicable than studies based solely on humans, and they can be validated by including human remains alongside the analogues (e.g. [14, 44]). Therefore, an adequate query is not whether we should abandon pig carcasses, but rather how pig carcasses and other animal models differ from human cadavers in certain aspects of their decomposition, for example, decomposition rate and patterns of colonisation by insects. Such research would put into perspective all the developments made possible over the past four decades by the use of human analogues (Table 1). Moreover, human cadavers are definitely limited resources for forensic sciences. Therefore, they should be invested to test hypotheses which were found to be forensically interesting for analogues, e.g. pig carcasses.

The need for robust replication and control are a direct consequence of both the inherent complexity of animal decomposition and the need for reliable forensic evidence in court. Our recommendations provide a quality assurance baseline for cadaver experiments. Indeed, simulated and reconstructed casework using pigs is an ideal test and cross-validation of conditions at a death scene (i.e. litigation research). Pig carcasses should be placed, if possible and acceptable, at or near the same site and time of year as the death scene and should serve as a reference for case analyses [67, 68].

A certain level of imprecision is inevitable even in superbly designed decomposition experiments, and court testimony will always need to draw cross-validation of decompositionbased estimates from other fields of science. Future decomposition studies will need to underpin their own importance with rigorous quality control measures $[27,28]$. A means to this end have been outlined here, and many of the recommendations apply as much to research with human corpses as to any other animal species.

Acknowledgements We thank David O. Carter (USA), Colin Moffatt (UK), Darryl J. de Ruiter (USA) and Amoret P. Whitaker (UK) for discussions and comments on earlier versions of the manuscript. Thanks are also extended to anonymous reviewers whose comments helped to improve the manuscript.

Open Access This article is distributed under the terms of the Creative Commons Attribution 4.0 International License (http:// creativecommons.org/licenses/by/4.0/), which permits unrestricted use, distribution, and reproduction in any medium, provided you give 
appropriate credit to the original author(s) and the source, provide a link to the Creative Commons license, and indicate if changes were made.

\section{References}

1. Kunkel J (2006) What makes a good model system. http://www.bio. umass.edu/biology/kunkel/modelsys.html. Accessed 25 Sep 2018

2. Zuk M, Garcia-Gonzalez F, Herberstein ME, Simmons LW (2014) Model systems, taxonomic bias, and sexual selection: beyond Drosophila. Annu Rev Entomol 59:321-338

3. Mégnin P (1894) La faune des cadavres, application de l'entomologie à la médecine légale. G. Masson, Paris

4. Williams KA, Villet MH (2006) A history of southern African research relevant to forensic entomology: review article. S Afr J Sci 102(1-2):59-65

5. Michaud JP, Schoenly KG, Moreau G (2015) Rewriting ecological succession history: did carrion ecologists get there first? Q Rev Biol 90(1):45-66

6. Payne JA (1965) A summer carrion study of the baby pig Sus scrofa Linnaeus. Ecology 46(5):592-602

7. Payne JA, King EW, Beinhart G (1968) Arthropod succession and decomposition of buried pigs. Nature 219(5159):1180-1181

8. Payne JA, King EW (1972) Insect succession and decomposition of pig carcasses in water. J Ga Entomol Soc 7(3):153-162

9. Catts EP, Goff ML (1992) Forensic entomology in criminal investigations. Annu Rev Entomol 37(1):253-272

10. Goff ML (2010) Early postmortem changes and stages of decomposition. In: Amendt J et al (eds) Current concepts in forensic entomology. Springer, Dordrecht, pp 1-24

11. Schoenly KG, Haskell NH, Mills DK, Bieme-Ndi C, Larsen K, Lee Y (2006) Recreating death's acre in the school yard: using pig carcasses as model corpses to teach concepts of forensic entomology \& ecological succession. Am Biol Teach 68(7):402-410

12. Schoenly KG (1992) A statistical analysis of successional patterns in carrion-arthropod assemblages: implications for forensic entomology and determination of the postmortem interval. J Forensic Sci 37(6): 1489-1513

13. Schoenly KG, Griest K, Rhine S (1991) An experimental field protocol for investigating the postmortem interval using multidisciplinary indicators. J Forensic Sci 36(5):1395-1415

14. Schoenly KG, Haskell NH, Hall RD, Gbur JR (2007) Comparative performance and complementarity of four sampling methods and arthropod preference tests from human and porcine remains at the forensic anthropology Center in Knoxville, Tennessee. J Med Entomol 44(5):881-894

15. Cadee GC (1991) The history of taphonomy. Columbia University Press, New York

16. Brain CK (1983) The hunters or the hunted?: an introduction to African cave taphonomy. University of Chicago Press, Chicago

17. Martin RE (1999) Taphonomy: a process approach, vol 4. Cambridge University Press

18. Beary MO, Lyman RL (2012) The use of taphonomy in forensic anthropology: past trends and future prospects. In: Dirkmaat D (ed) A companion to forensic anthropology. Wiley, pp 499-527

19. Forbes S (2017) Body farms. Forensic Sci Med Pathol 13(4):477479

20. Wallman JF (2017) Body farms. Forensic Sci Med Pathol 13(4): 487-489

21. Oostra RJ (2018) The making of a human taphonomy facility in Amsterdam, the Netherlands. In: 15th meeting of the European Association for Forensic Entomology, abstract book
22. Contributors (2018) Body farm. Wikipedia. The free encyclopedia. https://en.wikipedia.org/wiki/Body farm. Accessed 30 June 2018

23. Connor M, Baigent C, Hansen ES (2018) Testing the use of pigs as human proxies in decomposition studies. J Forensic Sci 63(5): $1350-1355$

24. Dautartas A, Kenyhercz MW, Vidoli GM, Meadows Jantz L, Mundorff A, Steadman DW (2018) Differential decomposition among pig, rabbit, and human remains. J Forensic Sci 63(6): 1673-1683

25. Adlam RE, Simmons T (2007) The effect of repeated physical disturbance on soft tissue decomposition-are taphonomic studies an accurate reflection of decomposition? J Forensic Sci 52(5): 1007-1014

26. Black S (2017) Body farms. Forensic Sci Med Pathol 13(4):475476

27. Bytheway JA, Connor M, Dabbs GR, Johnston CA, Sunkel M (2015) The ethics and best practices of human decomposition facilities in the United States. Forensic Sci Policy Management: An International Journal 6(3-4):59-68

28. Tomberlin J, Byrd J, Wallace J, Benbow M (2012) Assessment of decomposition studies indicates need for standardized and repeatable research methods in forensic entomology. J Forensic Res 3:147

29. Ericsson AC, Crim MJ, Franklin CL (2013) A brief history of animal modeling. Mo Med 110(3):201

30. Lieschke GJ, Currie PD (2007) Animal models of human disease: zebrafish swim into view. Nat Rev Genet 8(5):353

31. Nestler EJ, Hyman SE (2010) Animal models of neuropsychiatric disorders. Nat Neurosci 13(10):1161

32. Pearce A, Richards R, Milz S, Schneider E, Pearce S (2007) Animal models for implant biomaterial research in bone: a review. Eur Cell Mater 13:1):1-1)10

33. Schoenly K, Reid W (1987) Dynamics of heterotrophic succession in carrion arthropod assemblages: discrete series or a continuum of change? Oecologia 73(2):192-202

34. Matuszewski S, Bajerlein D, Konwerski S, Szpila K (2011) Insect succession and carrion decomposition in selected forests of Central Europe. Part 3: succession of carrion fauna. Forensic Sci Int 207(13):150-163

35. Villet MH (2011) African carrion ecosystems and their insect communities in relation to forensic entomology. Pest Technol 5(1):1-15

36. Henssge $\mathrm{C}$ (2016) Basics and application of the 'nomogram method' at the scene. In: Madea B (ed) Estimation of the time since death. CRC Press, Boca Raton, pp 63-114

37. Schoenly KG, Michaud JP, Moreau G (2016) Design and analysis of field studies in carrion ecology. In: Benbow ME, Tomberlin JK, Tarone AM (eds) Carrion ecology, evolution, and their applications. CRC Press, Boca Raton, pp 129-148

38. Schoenly KG, Shahid SA, Haskell NH, Hall RD (2005) Does carcass enrichment alter community structure of predaceous and parasitic arthropods? A second test of the arthropod saturation hypothesis at the anthropology research facility in Knoxville, Tennessee. J Forensic Sci 50(1):134-142

39. Shahid SA, Schoenly K, Haskell NH, Hall RD, Zhang W (2003) Carcass enrichment does not alter decay rates or arthropod community structure: a test of the arthropod saturation hypothesis at the anthropology research facility in Knoxville, Tennessee. J Med Entomol 40(4):559-569

40. Damann FE, Tanittaisong A, Carter DO (2012) Potential carcass enrichment of the University of Tennessee Anthropology Research Facility: a baseline survey of edaphic features. Forensic Sci Int 222(1):4-10

41. Metcalf JL, Wegener Parfrey L, Gonzalez A, Lauber CL, Knights D, Ackermann G, Humphrey GC, Gebert MJ, Van Treuren W, Berg-Lyons D, Keepers K, Guo Y, Bullard J, Fierer N, Carter DO, Knight R (2013) A microbial clock provides an accurate estimate of the postmortem interval in a mouse model system. eLife 2:e01104 
42. Metcalf JL, Xu ZZ, Weiss S, Lax S, Van Treuren W, Hyde ER, Song SJ, Amir A, Larsen P, Sangwan N, Haarmann D, Humphrey GC, Ackermann G, Thompson LR, Lauber C, Bibat A, Nicholas C, Gebert MJ, Petrosino JF, Reed SC, Gilbert JA, Lynne AM, Bucheli SR, Carter DO, Knight R (2016) Microbial community assembly and metabolic function during mammalian corpse decomposition. Science 351(6269): 158-162

43. Pechal JL, Crippen TL, Benbow ME, Tarone AM, Dowd S, Tomberlin JK (2014) The potential use of bacterial community succession in forensics as described by high throughput metagenomic sequencing. Int J Legal Med 128(1):193-205

44. Wang Y, Ma MY, Jiang XY, Wang JF, Li LL, Yin XJ, Wang M, Lai Y, Tao LY (2017) Insect succession on remains of human and animals in Shenzhen, China. Forensic Sci Int 271:75-86

45. Matuszewski S, Fratczak K, Konwerski S, Bajerlein D, Szpila K, Jarmusz M, Szafalowicz M, Grzywacz A, Madra A (2016) Effect of body mass and clothing on carrion entomofauna. Int J Legal Med 130(1):221-232

46. Whitaker AP (2014) Development of blowflies (Diptera: Calliphoridae) on pig and human cadavers - implications for forensic entomology casework. PhD Thesis, King's College, London, UK

47. Dekeirsschieter J, Frederickx C, Verheggen FJ, Boxho P, Haubruge E (2013) Forensic entomology investigations from doctor Marcel Leclercq (1924-2008): a review of cases from 1969 to 2005. J Med Entomol 50(5):935-954

48. Charabidze D, Colard T, Vincent B, Pasquerault T, Hedouin V (2014) Involvement of larder beetles (Coleoptera: Dermestidae) on human cadavers: a review of 81 forensic cases. Int J Legal Med 128(6):1021-1030

49. Watson EJ, Carlton CE (2005) Insect succession and decomposition of wildlife carcasses during fall and winter in Louisiana. J Med Entomol 42(2): 193-203

50. Watson EJ, Carlton CE (2003) Spring succession of necrophilous insects on wildlife carcasses in Louisiana. J Med Entomol 40(3): 338-347

51. Megyesi MS, Nawrocki SP, Haskell NH (2005) Using accumulated degree-days to estimate the postmortem interval from decomposed human remains. J Forensic Sci 50(3):618-626

52. Quinn G, Keough M (2002) Experimental design and data analysis for biologists. Cambridge University Press, Cambridge

53. Mann RW, Bass WM, Meadows L (1990) Time since death and decomposition of the human body: variables and observations in case and experimental field studies. J Forensic Sci 35(1):103-111

54. Roberts LG, Spencer JR, Dabbs GR (2017) The effect of body mass on outdoor adult human decomposition. J Forensic Sci 62(5):11451150

55. Komar D, Beattie O (1998) Effects of carcass size on decay rates of shade and sun exposed carrion. J Can Soc Forensic Sci 31(1):35-43

56. Simmons T, Adlam RE, Moffatt C (2010) Debugging decomposition data-comparative taphonomic studies and the influence of insects and carcass size on decomposition rate. J Forensic Sci 55(1):8-13

57. Spicka A, Johnson R, Bushing J, Higley LG, Carter DO (2011) Carcass mass can influence rate of decomposition and release of ninhydrin-reactive nitrogen into gravesoil. Forensic Sci Int 209(13):80-85

58. Sutherland A, Myburgh J, Steyn M, Becker PJ (2013) The effect of body size on the rate of decomposition in a temperate region of South Africa. Forensic Sci Int 231(1-3):257-262

59. Matuszewski S, Konwerski S, Fratczak K, Szafalowicz M (2014) Effect of body mass and clothing on decomposition of pig carcasses. Int J Legal Med 128(6):1039-1048

60. Perez AE, Haskell NH, Wells JD (2016) Commonly used intercarcass distances appear to be sufficient to ensure independence of carrion insect succession pattern. Ann Entomol Soc Am 109(1):72-80

61. Moreau G, Michaud J-P, Schoenly KG (2015) Experimental design, inferential statistics and computer modeling. In: Forensic entomology international dimensions and frontiers contemporary topics in entomology. CRC Press, Taylor \& Francis, Boca Raton, pp 205230

62. Tessmer J, Meek C (1996) Dispersal and distribution of Calliphoridae (Diptera) immatures from animal carcasses in southern Louisiana. J Med Entomol 33(4):665-669

63. Lewis A, Benbow M (2011) When entomological evidence crawls away: Phormia regina en masse larval dispersal. J Med Entomol 48(6):1112-1119

64. Roberts LG, Dabbs GR (2015) A taphonomic study exploring the differences in decomposition rate and manner between frozen and never frozen domestic pigs (Sus scrofa). J Forensic Sci 60(3):588594

65. Keough N, Myburgh J, Steyn M (2017) Scoring of decomposition: a proposed amendment to the method when using a pig model for human studies. J Forensic Sci 62(4):986-993

66. Wells J, LaMotte L (2017) The role of a PMI-prediction model in evaluating forensic entomology experimental design, the importance of covariates, and the utility of response variables for estimating time since death. Insects 8(2):47

67. Faucherre J, Cherix D, Wyss C (1999) Behavior of Calliphora vicina (Diptera, Calliphoridae) under extreme conditions. J Insect Behav 12(5):687-690

68. Turner B, Wiltshire P (1999) Experimental validation of forensic evidence: a study of the decomposition of buried pigs in a heavy clay soil. Forensic Sci Int 101(2):113-122

Publisher's note Springer Nature remains neutral with regard to jurisdictional claims in published maps and institutional affiliations. 